\title{
Scheduling EURO-k Conferences
}

\section{Stidsen, Thomas Jacob Riis; Pisinger, David; Vigo, Daniele}

\section{Published in:}

European Journal of Operational Research

Link to article, DOI:

10.1016/j.ejor.2017.10.015

Publication date:

2018

Document Version

Peer reviewed version

Link back to DTU Orbit

\section{Citation (APA):}

Stidsen, T. J. R., Pisinger, D., \& Vigo, D. (2018). Scheduling EURO-k Conferences. European Journal of Operational Research, 270(3), 1138-1147. https://doi.org/10.1016/j.ejor.2017.10.015

\section{General rights}

Copyright and moral rights for the publications made accessible in the public portal are retained by the authors and/or other copyright owners and it is a condition of accessing publications that users recognise and abide by the legal requirements associated with these rights.

- Users may download and print one copy of any publication from the public portal for the purpose of private study or research.

- You may not further distribute the material or use it for any profit-making activity or commercial gain

- You may freely distribute the URL identifying the publication in the public portal

If you believe that this document breaches copyright please contact us providing details, and we will remove access to the work immediately and investigate your claim 


\section{Accepted Manuscript}

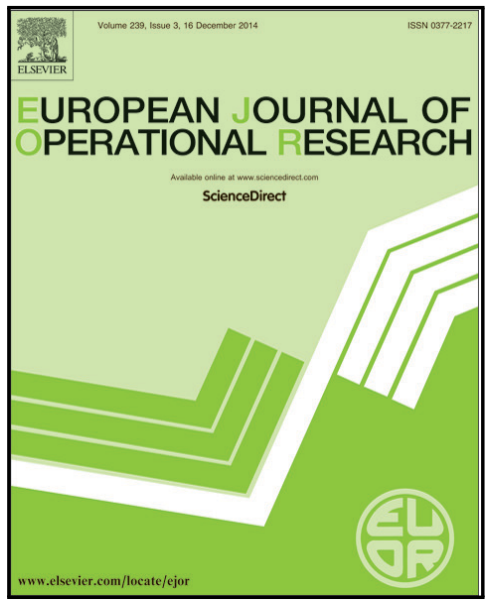

Scheduling EURO-k Conferences

Thomas Stidsen, David Pisinger, Daniele Vigo

PII:

S0377-2217(17)30928-1

DOI:

10.1016/j.ejor.2017.10.015

Reference:

EOR 14739

To appear in:

European Journal of Operational Research

Received date:

7 November 2016

Revised date:

30 September 2017

Accepted date:

10 October 2017

Please cite this article as: Thomas Stidsen, David Pisinger, Daniele Vigo, Scheduling EURO-k Conferences, European Journal of Operational Research (2017), doi: 10.1016/j.ejor.2017.10.015

This is a PDF file of an unedited manuscript that has been accepted for publication. As a service to our customers we are providing this early version of the manuscript. The manuscript will undergo copyediting, typesetting, and review of the resulting proof before it is published in its final form. Please note that during the production process errors may be discovered which could affect the content, and all legal disclaimers that apply to the journal pertain. 


\title{
Scheduling EURO-k Conferences
}

\author{
Thomas Stidsen ${ }^{\mathrm{a}, *}$, David Pisinger ${ }^{\mathrm{a}}$, Daniele Vigo ${ }^{\mathrm{b}}$ \\ a Technical University of Denmark \\ ${ }^{b}$ DEI "Guglielmo Marconi" - University of Bologna, Italy
}

\begin{abstract}
EURO-k conferences are among the largest Operations Research conferences in the world, typically including more than 2000 presentations. As opposed to many other conferences, EURO-k conferences are hierarchically organized, and the conference schedule should reflect this structure to make navigation easier and more logical. In this/article we present a scheduling tool that has been developed during the EURO2015 and EURO2016 conferences to schedule the streams, sessions and talks. A schedule is obtained by solving a number of optimization models, each addressing a specific objective. First, areas are assigned to buildings, making sure that related research areas are located close to each other. Next, the goal is to allocate each stream to only one room, and to ensure that the stream consists of a sequence of consecutive time slots. Finally, we optimize the assignment of room sizes. We illustrate the process by showing results from the scheduling of the EURO2016 conference, which took place in Poznan (Poland), July 3-6, 2016.
\end{abstract}

Keywords: Project Scheduling, Conference scheduling, Mixed Integer Programming

\section{Introduction}

The EURO-k conferences are the largest Operations Research (OR) conferences in Europe, and among the largest worldwide. Each conference is hosted by one of the member societies of EURO, the Association of European Operational Research Societies, and typically attracts over 2000 participants from all over the world. The EURO-k conferences are held annually, except when the conference of the International Federation of Operational Research Societies (IFORS) conference is held in the same year.

A significant amount of scheduling activities is necessary behind such a large conference and a number of people in the program and organizing committees work for months ahead of the eonference. Luckily, Operations Research offers advanced scheduling tools for some of these tasks. In this article we describe how the use of Mixed Integer Programming (MIP) models supports the scheduling of the time-slots and rooms allocated to the different sessions.

\footnotetext{
*Corresponding author: Thomas Stidsen, Building 426, Produktionstorvet 2800 Kgs. Lyngby, Denmark

Email addresses: thst@dtu.dk (Thomas Stidsen), dapi@dtu.dk (David Pisinger), daniele.vigo@unibo.it (Daniele Vigo)
} 
Due to the large number of presentations and diversity of subjects, EURO-k conferences are organized hierarchically. Each EURO-k conference consists of a number of areas (i.e., themes) that are defined by the program committee in order to group presentations that are homogeneous with respect to either the methodological content or the application area. The program committee assigns the overall organization and monitoring of each area to one or more of its members. The area responsibles in turn invite a number of key researchers or EURO working group managers to organize streams (i.e., groups of sessions) within the area topic. Finally, the stream organizers invite other researchers to organize one or more sessions, each consisting of four talks. The session organizers are frequently asked to chair the session they have organized in order to acknowledge their efforts. The abstracts of invited papers are reviewed by stream and area responsibles, and those of acceptable standard are included in the conference program. Because of the hierarchical organization, the scheduling process in this paper is about assigning day, timeslot and room to the sessions. The actual ordering of sessions within a stream and of talks within the sessions is left to the stream and session organizers to ensure that they can adjust the stream schedule to the specific need of the stream and the speakers, leaving them some control on the lower level of the program.

In parallel to the hierarchy of invited talks, participants are also allowed to submit a contributed talk to the conference within one of the conference areas. The contributed talks constitute around one third of the submissions, and they go through the same review process as the invited talks. Contributed talks are frequently used to fill holes in invited sessions or they are grouped into new sessions according to a common topic and associated with existing streams. As such, there is no real difference between invited talks and contributed talks and sessions in the final program.

A typical EURO-k conference with 1800-2000 talks includes about 5-600 sessions: due to last-minute cancellations and other program requirements, not all sessions have the standard number of four talks. The sessions are arranged in 100-150 streams belonging to about 25 areas. In the conference program, the sessions are scheduled in time slots of 90 minutes that allow about 20 minutes for each presentation and some minutes devoted to questions and discussion. Depending on the specific conference calendar there are 10 or 11 time slots available across three days. As a consequence, between 40 and 60 sessions should be assigned to each time slot in parallel.

In order to enable a clear and easy to understand conference program, it is generally desirable that the program of a EURO-k conference follows the hierarchical structure of the invited talks. This means that main areas which are related to each other (such as, "Routing, Location, Logistics and Transportation" and "Production Management and Supply Chain Management") should be scheduled in the same building (or in nearby ones) such that participants interested in the research field easily can move quickly between the talks. Also, each stream should ideally consist of a sequence of consecutive sessions in the conference time slots, all assigned to the same room throughout the conference. However, if a stream consists of more sessions than the available number of time slots, then it may be necessary to split the stream into two or more parts.

As a rule of thumb, a speaker is only allowed to give one presentation during a EURO$\mathrm{k}$ conference. A few exceptions exists to this rule. This may include talks in "OR and 
teaching", workshops or other similar activities. The main speaker of each talk needs to register to the conference, otherwise the talk is cancelled and deleted. A delegate can be co-author of as many talks as needed, but due to the imposed complexity it cannot be guaranteed that there will be no overlap in the schedule between the co-authored talks.

Table 1 shows a list of the 25 different areas for EURO2016. Each area consists of a number of different streams, resulting in a total of 124 streams in 2016. For the sake of brevity we refrain from listing all the streams. Each stream consists of a number of sessions, in each of which up to four presentations are given. Each stream has a designated organizer who is in charge of the detailed scheduling of which talk is given in which session. The stream organizers are responsible for defining a proper ordering of the sessions, and session organizers should order the talks in their sessions.

\subsection{Previous work}

Conference scheduling has been quite well studied as an optimization problem and in [1] we find a detailed survey of the problem. The survey distinguishes between a presenter-based perspective, which strives to avoid clashes between presenter duties, and a attendee-based perspective, which strives to make the conference easily accessible for the attendees. Besides the articles surveyed in [1], we would like to also mention the following results on conference scheduling $[2,3,4,5]$.

In [4] a MIP model is presented for preference-based conference scheduling. The model maximizes a general participant utility function while ensuring solution feasibility. The model is solved using a simulated annealing algorithm. The scheduling tool has been applied to a conference with 213 sessions over 10 time-blocks invovling 1086 attendees.

The papers $[2,5]$ present a community-informed conference scheduling tool called Cobi. Cobi comprises community sourcing applications for collecting preferences and constraints from community members, and a visual scheduling interface that enables the organizers to make informed improvements to the schedule. The scheduling is done in two steps: First, committee members are invited to group papers in their areas of expertise, and next authors of the accepted papers are invited to identify papers that would complement their own within the same session. The tool has been used for a number of large conferences involving more than 1000 authors and 3000 attendees.

The conference scheduling tool Confer is presented in [3]. Confer helps conference attendees find interesting papers and talks and discover people with shared interests, while providing them with a personalized schedule for the conference. The tool makes use of collaborative filtering to provide social recommendations. Confer was applied to the scheduling of the CSCW 2016 conference.

Scheduling of EURO-k conferences differs from most of these papers in that the main objective is to follow the hierarchical structure of the conference, together with the fact that EURO is significantly larger than the previously scheduled conferences. However, in the future it could be extended to handle time clashes between popular talks or to ensure a fair attendance to all sessions. The main reason for circumventing these criteria in the present paper is that currently no data is available about listener wishes, nor about the expected number of attendees to talks. Obtaining such preferences from the registered participants 
before the scheduling would require a complete redesign of the conference registration system, which was not possible at the time of scheduling. Furthermore, unfortunately no reliable data about the actual number of attendees of each session is available from previous EURO conferences. It is clear that, whenever such information will be available in the future it may positively influence the overall quality of the result without the need to change the structure of our approach.

There are a number of software packages supporting Conference Administration. To the best of our knowledge, however, they do not consider the more strategic approach where the detailed scheduling of the presentations in sessions is left to the stream organizer.

\subsection{Outline}

The main contribution of this paper is to present a multi-objective MIP model for scheduling the EURO-k conference that supports the hierarchical structure of the conference. To the best of our knowledge, no mathematical model has been presented that tackles the problem from this viewpoint. A second contribution is to handle the many criteria in the objective, and to leave sufficient flexibility to stream organizers.

In Section 2, we describe the scheduling process in detail and Section 3 we show a concrete example - the EURO2016 conference. In Section 4 we discuss which criteria should be used to define a good schedule, while we in Section 5 describe the MIP model. The model is solved in a number of steps, each optimizing one of the main criteria described in the preceding section. In Section 6 we present the results for EURO2016 and discuss benefits of using the model. In Section 7 we discuss challenges and future improvements. Finally we conclude the paper in Section 8 by discussing possible future improvements.

The approach used in this article is a further development of the approach used for the EURO2015 conference in Glasgow. We note that even though the scheduling methodology described in this paper is developed explicitly for EURO-k conferences, the structure of areas/stream/session is quite common, at least in scientific conferences. Moreover, similar hierarchical structures are also common in other scheduling problems such as educational timetabling. We therefore think that many features of the methods described in what follows can be easily adapted to other contexts.

\section{The Scheduling Process}

The scheduling tool should only generate a high-level schedule, where each stream is allocated to a/specific room and to a set of possibly consecutive time slots. We do not generate a low-level schedule for several reasons: First of all, it should be left to the stream organizers to schedule the individual sessions and talks in a logical order as described in Section 4. Moreover, a low-level schedule would demand much more detailed data than what is presently available.

As already mentioned, it is indeed the responsibility of the stream organizers to adjust the assignment of the talks to sessions, and to order the sessions in the stream according to various criteria. For instance, a session on a given topic could start with a talk defining the problem and discussing modeling issues, while subsequent talks could go in depth into 


\begin{tabular}{|c|c|c|}
\hline Area name & $\begin{array}{r}\text { Number of } \\
\text { streams }\end{array}$ & $\begin{array}{r}\text { Number of } \\
\text { sessions }\end{array}$ \\
\hline Analytics, Data Science and Data Mining & 4 & 21 \\
\hline Artificial Intelligence, Fuzzy Systems and Computing & 3 & 7 \\
\hline Continuous Optimization & 7 & 28 \\
\hline Control Theory and System Dynamics & 6 & 17 \\
\hline Decision Analysis, Decision Support Systems, DEA and Performance Measurement & 4 & 12 \\
\hline Discrete Optimization, Mixed Integer Linear and Nonlinear Programming & 5 & 30 \\
\hline Emerging Applications of OR & 10 & 18 \\
\hline Energy, Environment, Natural Resources and Climate & 6 & 18 \\
\hline Financial Modeling, Risk Management and Managerial Accounting & 9 & 24 \\
\hline Game Theory and Mathematical Economics & 5 & 11 \\
\hline Graphs and Networks & & \\
\hline Metaheuristics & & 6 \\
\hline Multiple Criteria Decision Making and Optimization & & 47 \\
\hline OR Education & & 5 \\
\hline OR for Developing Countries and Humanitarian Applications & & 10 \\
\hline OR History and OR Ethics & 3 & 7 \\
\hline OR in Health, Life Sciences and Sports & 8 & 31 \\
\hline OR in Industry and Software for OR & 6 & 13 \\
\hline Practice of OR (Making an Impact) & 3 & 8 \\
\hline Production Management and Supply Chain Management & 6 & 26 \\
\hline Revenue Management & 1 & 2 \\
\hline Routing, Location, Logistics and Transportation & 9 & 53 \\
\hline Scheduling, Timetabling and Project Management & 6 & 29 \\
\hline Simulation, Stochastic and Robust Optimization & 2 & 10 \\
\hline Soft OR, Problem Structuring Methods and Behavioural OR & 2 & 13 \\
\hline Total & 124 & 463 \\
\hline
\end{tabular}

Table 1: Research areas at EURO2016. Each area can be regarded as a mini-conference in the EURO conference, hence its sessions should be located close to each other

the solution methods in a progressive way. Similarly, sessions introducing methodological advances should precede or follow sessions discussing application issues in a specific field.

Speakers may have various wishes with respect to the scheduling of their talks. This could include either having to leave early the last day, or arriving late the first day, or having to attend a session or one of the numerous business meetings during the conference at a specific time.

The submission system does not support such wishes, since making the facility available to all speakers could make the scheduling inextricable or lead to splitting of sessions and streams. However, speakers may contact their stream organizer in urgent situations, who can take them into account primarily by sorting the sessions accordingly. Since the stream organizer is doing the detailed scheduling of the talks in the stream, many conflicts and soft constraints can be handled by simply swapping the order of the speakers in a session, or reordering the sessions. If a speaker has to leave earlier the last day, the talk can be scheduled in the beginning of the stream. This works well for large streams with a large number of sessions, but has limited effect if the stream only has a few sessions. In that case the constraints need to be handled at the high-level scheduling by the scheduling team.

The submission system includes a tool for conflict detection that can identify if a speaker has two activities scheduled at the same time. This could include the speaker having to give a talk in one session while chairing another session assigned to the same time slot. Due to the rule of only having one presentation per delegate, the number of conflicts is generally 
low, and can easily be handled manually.

Since the exact sessions each delegate wants to participate in is not known, it is assumed that if a stream contains many talks, then many delegates will follow this stream. The room size for each stream should therefore be proportional to the number of talks in the whole stream (which may consist of many sessions). The rules of thumb may be overridden by the scheduling team, e.g., when it is known that a specific talk or session will attract a large audience.

\section{A concrete example: the EURO2016 Conference}

The EURO2016 conference was held in Poznan, Poland, July 3-6, 2016. More than 2000 researchers attended the conference and more than 1600 presented their work in one of the 463 sessions. The first day of the conference, Sunday 3rd of July, was only used for the opening session and registration. The master schedule for the remaining three days, Monday 4th to Wednesday 6th of July is given in table 2.

\begin{tabular}{|l|ccc|}
\hline Timeslot & Monday 4/7 & Tuesday $5 / 7$ & Wednesday $6 / 7$ \\
\hline Morning A & MA-08:30-10:00 & TA-08:30-10:00 & WA-08:30-10:00 \\
Morning B & MB-10:30-12:00 & TB-10:30-12:00 & WB-10:30-12:00 \\
Midday C & MC-12:30-14:00 & TC-12:30-14:00 & WC-12:30-14:00 \\
Afternoon D & MD-14:30-16:00 & TD-14:30-16:00 & TD-14:30-16:00 (PLENARY) \\
Afternoon E & ME-16:30-17:30 (PLENARY) & TE-16:00-17:45 (PLENARY) & WE-16:30-17:30 (CLOSING SESSION) \\
\hline
\end{tabular}

Table 2: Master schedule of EURO2016

The schedule has 11 time slots each with 90 minutes available for presentations. Time slots are identified as MA, MB, MC, MD, FA, TB, TC, TD, WA, WB, and WC where the first letter of the identifier clearly denotes the day. In each session there will typically be 4 presentations, but the final decision of how many presentations and which presentations is left to the stream organizer and the program committee. In Poznan, the venue of the EURO2016 conference was the Lecture and Conference Centre at the Poznan University of Technology. Below in Figure 1 the five buildings at the Lecture and Conference Centre are shown schematically and denoted as CW, BT, BM, WE and L. Each building contains a number of rooms for presentations as listed in table 3, and in total there were 54 rooms available.

\begin{tabular}{|c|cccccccccccccccc|c|c|}
\hline \\
Building & 1 & 2 & 3 & 4 & 5 & 6 & 7 & 8 & 9 & 10 & 11 & 12 & 13 & 14 & 15 \\
\hline CW & 665 & 146 & 84 & 59 & 146 & 200 & 200 & 146 & 72 & 200 & - & - & - & - & - \\
BT & 60 & 81 & 96 & 60 & 30 & 40 & 46 & 48 & 76 & 54 & 55 & 64 & 33 & - & - \\
BM & 60 & 60 & 30 & 60 & 40 & 40 & 66 & 35 & 16 & 40 & 60 & 60 & 60 & 15 & 30 \\
WE & 42 & 42 & 90 & 60 & 30 & 90 & 90 & 40 & 36 & 60 & 90 & 24 & - & - & - \\
L & 126 & 120 & 155 & 222 & - & - & - & - & - & - & - & - & - & - & - \\
\hline
\end{tabular}

Table 3: A Available buildings and rooms for EURO2016. The entries indicate the room size (number of seats). For simplicity, the rooms are numbered $1,2, \ldots$ instead of using the original names.

Given the set of available buildings, rooms and time slots, the scheduling task is to assign all the sessions of the different streams to time slots and rooms. 


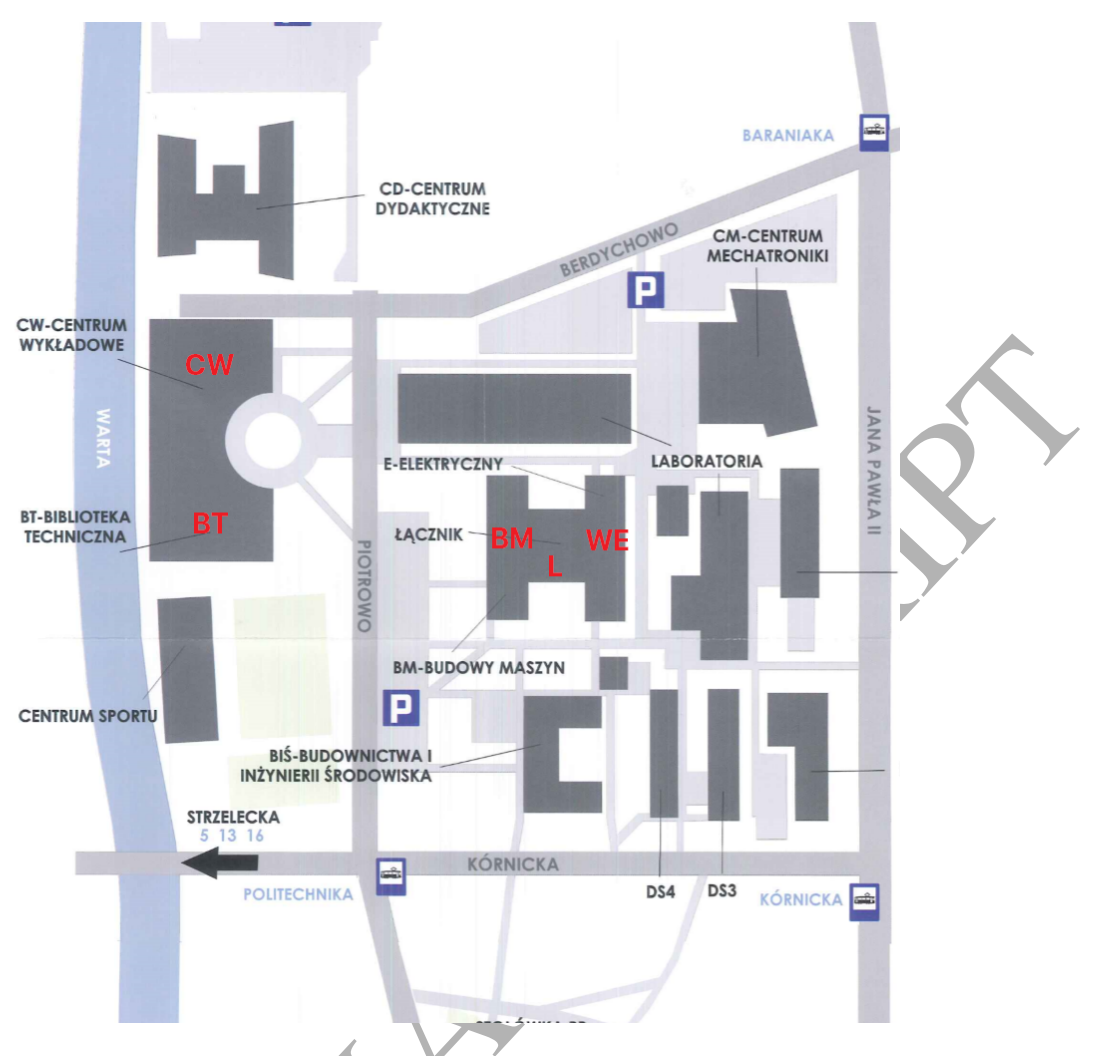

Figure 1: Lecture and Conference Centre, Poznan University of Technology, venue of EURO2016

\section{What is a good schedule?}

A good schedule should make it easy for conference attendees to participate in the sessions which are most important to them. Unfortunately, when the plan is made, the planners do not know which presentations each of the attendees wants to hear. Instead the planners will make a number of assumptions:

1. Attendees prefer to sit during the presentations, i.e., the room should be sufficiently large such that all attendees can sit, hence the room size should at least be as big as the number of speakers in a stream.

2. Attendees will concentrate their attention to a few streams, hence:

(a) Sessions in the same stream should not take place at the same time. This is a hard requirement of the scheduling.

(b) Sessions in the same stream should take place in the same building. This is a hard requirement.

(c) Sessions in the same stream should take place in the same room. This is a strong preference but not a hard requirement.

3. The streams an attendee is interested in is likely to be in the same area, hence each area should be in one building, if possible. 
4. Some of the areas are subject-related, so attendees that have an interest in streams in one area are likely to have an interest in a related area, and related pairs of areas should therefore be placed in the same building, if possible.

We acknowledge, that the above assumptions can be debated, in particular the assumptions about which sessions an attendee will follow. However, these assumptions are only used to define parameters in the model, and if better estimates can be obtained in future conferences, these estimates can be used as input.

In the models below we will aim at satisfying the assumptions by defining appropriate constraints and objective functions.

\section{MIP Model}

In this section we will formally formulate the session scheduling problem. We first give an overview of the sets, parameters, and variables.

\subsection{Sets}

$a \in \mathcal{A}$ : The set of areas

$s \in \mathcal{S}$ : The set of streams

$s \in \mathcal{S}_{a}$ : The subset of streams belonging to an area $a$

$b \in \mathcal{B}=\{C W, B T, B M, W E, L\}:$ Theset of buildings

$r \in \mathcal{R}_{b}$ : The set of rooms in building $b$

$d \in \mathcal{D}=\{M, T, W\}:$ The set of days

$t \in \mathcal{T}=\{A, B, C, D, E\}:$ The set of time slots per day

\subsection{Parameters}

$N_{s}$ : Number of sessions for stream $s$

$P_{s}$ : Number of people potentially attending stream $s$

$I_{a, a^{\prime}}$ : Incidence matrix which is 1 for distinct area pairs $\left(a, a^{\prime}\right)$ that are related

$\tilde{I}=\sum_{a, a^{\prime}} I_{a, a^{\prime}}$ : Number of pairs $\left(a, a^{\prime}\right)$ that need to be co-located.

$V A L_{d, t}$ : Unique identifier of the time slot $(d, t)$ in the range 1 to 11 in order of time. We here only consider the 11 time slots in which there are sessions, not the plenary time slots nor the time slot with the closing session.

$M=11$ The total number of timeslots for the whole conference MA, MB, MC, MD, TA, TB, TC, TD, WA, WB, and WC

$R S_{b, r}$ : Number of seats of room $r$ in building $b$.

utility $y_{a, s, b, r}=\log \left(\frac{R S_{b, r}-P_{s}}{P_{s}}+1\right)$. The utility value of a certain stream $s$ of area $a$, using a certain room $r$ is a positive number for all the rooms which can be assigned (see (1)), which grows with the number of extra seats in the room. The utility parameter rewards the number of extra seats for each session-room combination, but with decreasing growth, such that it is best to assign smaller streams to smaller rooms and larger streams to larger rooms. 


\subsubsection{Decision variables}

$x_{b, r, d, t}^{a, s} \in\{0,1\}: 1$ if a session of a stream $s$ of area $a$ is allocated to room $r$ in building $b$ on day $d$ at time slot $t$

$y_{b}^{a, s} \in\{0,1\}: 1$ if stream $s$ of area $a$ is allocated to a room in building $b$ $q_{a, b} \in\{0,1\}: 1$ if area $a$ use one or more rooms in building $b$ $v_{a, a^{\prime}, b} \in\{0,1\} 1$ if area $a$ and area $a^{\prime}$ are both in building $b$ $u_{b, r}^{a, s} \in\{0,1\}: 1$ if stream $s$ of area $a$ use room $r$ in building $b$

first ${ }^{a, s} \in \mathbb{R}^{+}$: The number of the first time slot for a session in stream $s$ of area $a$. Because there are 11 time slots, we associate them with increasing numbers from the first MA, with value 1 , to the last $\mathrm{WC}$, with value 11 .

last $^{a, s} \in \mathbb{R}^{+}$: The number of the last time slot for a session in stream $s$ of area $a$.

\subsubsection{Constraints}

Having defined all relevant variables and parameters, we are now able to write up the constraints of the model:

$$
\begin{array}{r}
x_{b, r, d, t}^{a, s}=0 \quad \forall a \in \mathcal{A}, s \in \mathcal{S}_{a}, b \in \mathcal{B}, r \in\left\{\mathcal{R}_{b} \mid R S_{b, r}>P_{s}\right\}, d \in \mathcal{D}, t \in \mathcal{T}(1) \\
\sum_{b \in \mathcal{B}} y_{b}^{a, s}=1 \quad \forall a \in \mathcal{A}, s \in \mathcal{S}_{a}
\end{array}
$$

Constraint (1) ensures the sessions of a stream are only allocated to rooms where there are enough seats. Constraint (2) ensures that all sessions of a stream take place in the 
same building. Constraint (3) ensures that at most one session takes place in each room in each building in each time slot each day. Constraint (4) ensures that all sessions for each stream gets allocated. Constraint (5) ensures that there are no parallel sessions for each stream. Constraint (6) ensures that variable $q_{a, b}$ is set to 1 if a stream of area $a$ use a room in a building. Constraint (7) ensures that variable $y_{b}^{a, s}$ is set to 1 if a stream $s$ of area $a$ uses a room in building $b$. Constraint (8) and Constraint (9) limits the $v_{a, a^{\prime}, b}$ variable. Constraint (10) ensures that the variable $u_{b, r}^{a, s}$ has the value 1 if stream $s$ of area $a$ use room $r$ in building $b$. Constraint (11) limits the highest value of first $^{a, s}$ to the value of the first time slot used by a session in stream $s$ of area $a$ and Constraint (12) limits the lowest value of $l_{a s t}{ }^{a, s}$ to the value of the last time slot used by a session in stream $s$ and area $a$.

\subsection{Objectives}

In Section 4 we discussed various requirements that need to be satisfiedin a good schedule of the conference. We will now describe how to reach this goal using five different objective functions. Finding the Pareto Front solutions to the five different objectives is unfortunately not possible by current techniques. Clearly several other techniques can be adopted, such as using a weighted objective function in which the weight of each criterion must be defined apriori. In our case, we adopted a lexicographic approach in which the different objectives are ranked according to their importance and solved sequentially. Our computational experience with this approach showed that we were able to find optimal or near-optimal solutions at each step when compared with the lower and upper bound on each objective values.

For all solutions we require that there are enough seats to the attendees (Constraint (1)), that all the sessions of a stream take place in the same building (Constraint (2)) and that there are no more than 1 session of a stream in a time slot (Constraint (5)).

Below we describe the five objectives we use:

- Objective 1: Minimize the number of areas which takes place in more than one building. A lower bound equal to 0 is obtained if all areas are in one building each.

- Objective 2: Maximize the number of co-located areas to the same building, if it is specified in the matrix $I_{a, a^{\prime}}$. An upper bound $\tilde{I}$ is obtained if all related pairs of areas are placed in the same building. Notice that we get as input the relations between the areas, i.e., the pairs of areas to be preferably co-located.

- Objective 3. Minimize the number of different rooms used for each stream, deducted by 1 for elach stream. A lower bound equal to 0 is obtained if each stream use just one room.

- Objective 4: Minimize the number of time gaps in the streams. A lower bound equal to 0 is obtained if all streams have consecutive sessions without holes.

- Objective 5: Maximize the residual room capacity.

As mentioned above, we solve five MIP models sequentially, using lexicographic optimization. 


\subsection{Step 1: Unique buildings to streams and areas}

The first step aims at minimizing the number of buildings used for the different areas, as formulated below in model (13). The requirement that there are enough seats and that all sessions in a stream are allocated to rooms in the same building is ensured by constraint (1) and constraint (2). The model will in the following be denoted MIP1:

$$
\min z_{1}=\sum_{a \in \mathcal{A}}\left(\left(\sum_{b \in \mathcal{B}} q_{a, b}\right)-1\right)
$$

$$
\begin{aligned}
& (1)-(7) \\
& q_{a, b}, y_{b}^{a, s}, x_{b, r, d, t}^{a, s} \in\{0,1\}
\end{aligned}
$$

\subsection{Step 2: Co-allocation of related areas}

Given an optimal solution to the Step 1 model of value $\bar{z}_{1}$ MIP2 (14) consists of the appropriate objective function, i.e. maximizing the area co-location, constrained by the lexicographic constraint from Step 1. To make the problem easier to solve, we help the solver by ruling out gains for co-location if areas are spread over more than one building, i.e., if $\forall a \mid \sum_{b} \bar{q}_{a, b}>1$. In that case we rule out gains for co-location, i.e., $v_{a, a^{\prime}, b}=0$.

$$
\begin{aligned}
& \max z_{2}=\sum_{a \in \mathcal{A}} \sum_{a^{\prime} \in \mathcal{A}} \sum_{b \in \mathcal{B}} I_{a, a^{\prime}} \cdot v_{a, a^{\prime}, b} \\
& \text { s.t. } \\
& \sum_{a \in \mathcal{A}}\left(\left(\sum_{b \in \mathcal{B}} q_{a, b}\right)-1\right) \leq z_{1} \\
& v_{a, a^{\prime}, b}=0 \quad \forall b, a \in\left\{\mathcal{A} \mid \sum_{b^{\prime} \in \mathcal{B}} \bar{q}_{a, b^{\prime}}>1\right\}, a^{\prime} \in\left\{\mathcal{A} \mid \sum_{b^{\prime} \in \mathcal{B}} \bar{q}_{a^{\prime}, b^{\prime}}>1\right\} \\
& (1)-(10) \\
& q_{a, b}, v_{a, a^{\prime}, b}, y_{b}^{a, s}, x_{b, r, d, t}^{a, s} \in\{0,1\}
\end{aligned}
$$

Notice, that because we now fix the areas to specific buildings, the following models decompose into onel model for each of the buildings. This means that in the following models MIP3, MIP4 and MIP5 the models are solved sequentially for each building. The result from MIP2, i.e. pairs $(a, s)$ for which $\bar{y}_{b}^{a, s}=1$, defines which streams $s$ have been placed in which building $b$. To ease notation, we define the auxillary set $H_{b}=\left\{(a, s) \in\left(\mathcal{A}, \mathcal{S}_{a}\right) \mid \bar{y}_{b}^{a, s}=1\right\}$. In the following models MIP3, MIP4 and MIP5 are optimized for each building $b$, and the objective functions and constraints are only defined for the set of streams $a, s$ which belong to the set $H_{b}$.

\subsection{Step 3: Room stability}

In MIP3 (15) we try to make it easier for the attendees to find the rooms of the sessions. This is done by minimizing the number of rooms used by each stream, such that attendees 
only have to find the room of the preferred stream once. To minimize the number of rooms, we need (a lot) more binary variables, i.e. $u_{b, r}^{a, s}$ variables. Hence, we perform a trick: We decompose the problem into different buildings, i.e., we solve 5 models, one for each building. This decomposition is possible because we constrain each stream to one building only (2), i.e. the one where $y_{b}^{a, s}=1$. Such decomposition could lead to bad performance, but the results are so close to the lower bound that it is acceptable.

$$
\begin{aligned}
& \min _{b} z_{3 b}=\sum_{(a, s) \in H_{b}} \sum_{r \in \mathcal{R}_{b}}\left(u_{b, r}^{a, s}-1\right) \\
& \text { s.t. } \\
& (1)-(10) \quad(a, s) \in H_{b} \\
& y_{b}^{a, s}, u_{b, r}^{a, s}, x_{b, r, d, t}^{a, s} \in\{0,1\}
\end{aligned}
$$

\subsection{Step 4: Gaps in stream minimizing}

In Step 4 we aim at making the sessions of a stream sequential, again making it easier for the attendee to follow a specific stream. The solution from Step 3 is carried over to Model (16) below, by instantiating a constraint which limits the number of rooms used to the same number as found in Step $3\left(\bar{z}_{3 b}\right)$.

$$
\begin{aligned}
& \min _{b} z_{4 b}=\sum_{(a, s) \in H_{b}}\left(\text { last }^{a, s}-\text { first }^{a, s}-N_{s}+1\right) \\
& \text { s.t. } \\
& \sum_{(a, s) \in H_{b}}\left(\left(\sum_{r \in \mathcal{R}_{b}} u_{b, p}^{a, s}\right)-1\right) \leq \bar{z}_{3 b} \\
& \text { (1) }-(12) \quad(a, s) \in H_{b} \\
& y_{b}^{a, s}, u_{b, r}^{a, s}, x_{b, r, d, t}^{a, s} \in\{0,1\} \\
& \text { first }{ }^{a, s}, \text { last }^{a, s} \in \mathbb{R}^{+}
\end{aligned}
$$

The model is denoted MIP4.

\subsubsection{Step5: Room utility maximization}

In the last step the aim is to choose rooms such that we maximize the probability that there are enough seats for each session. In the previous models we have ensured through Constraint (1) that only rooms with enough seats are chosen for the sessions. The number of attendees $P_{s}$ to a session is estimated from the number of presentations in a stream. This is obviously an assumption which is highly likely to be wrong. Hence we want to plan such that the distribution of extra seats is well distributed. We utilize a utility factor utility $y_{a, s, b, r}$ which should make sessions be allocated such that smaller sessions will use smaller rooms and larger sessions will use larger rooms. 


$$
\begin{aligned}
& \max _{b} z_{5 b}=\sum_{(a, s) \in H_{b}} \sum_{r \in \mathcal{R}_{b}} \sum_{t \in \mathcal{T}} \text { utility }_{a, s, b, r} \cdot x_{b, r, d, t}^{a, s} \\
& \text { s.t. } \\
& \sum_{(a, s) \in H_{b}}\left(\text { last }^{a, s}-\text { first }^{a, s}-N_{s}+1\right) \leq \bar{z}_{4 b} \\
& \sum_{(a, s) \in H_{b}} \sum_{r \in \mathcal{R}_{b}} u_{b, r}^{a, s} \leq \bar{z}_{3 b} \\
& (1)-(12) \quad(a, s) \in H_{b} \\
& y_{b}^{a, s}, u_{b, r}^{a, s}, x_{b, r, d, t}^{a, s} \in\{0,1\} \\
& \text { first }^{a, s}, \text { last }^{a, s} \in \mathbb{R}^{+}
\end{aligned}
$$

This, last, optimization model is denoted MIP5.

\section{Result}

The 5 different MIP models MIP1 to MIP5 were implemented in GAMS Ver. 24.3.3 and solved using CPLEX Ver. 12.6. The scheduling of EURO2016 took place in the days May 9-19, 2016. The data behind the scheduling are available from [6] as an excel file and an accompanying explanation word file. Because the whole solution time of models MIP1 to MIP5 is limited to a few hours, it became possible to solve the models a number of times with different inputs. This enabled planners to alter the input in several ways:

- Changes in the number of attendees and presentations

- Removal of the smallest rooms

- Removal of Second floor rooms of the buildings WE and BM, due to lack of air condition in these rooms

In the final iteration we found the following objective values for the five steps:

- In MIP1 we find the optimal solution value $\bar{z}_{1}=0$, meaning that no area needs more than one building. The trivial lower bound for the objective value is 0 , so the solution cannot be improved.

- In the next step, MIP2 maximizes the total number of pairs of areas for which colocation is requested. The upper bound was $\bar{I}=37$ for EURO2016. The optimal solution to MIP2 was $\bar{z}_{2}=33$, which is quite close to the upper bound.

- MIP3 minimizes the number of different rooms used for a given stream. The optimal solution value is found to be $\sum_{b} \bar{z}_{3 b}=0$, meaning that each stream is assigned to only one room, and no room shifts are necessary. The solution matches the lower bound and can therefore not be improved. 
- Next, MIP4 minimizes the number of time gaps in each stream. We find the solution $\bar{z}_{4 b}=0$ for all buildings, except building BT where the gap is $\bar{z}_{4 b}=2$. The trivial lower bound is 0 , which is obtained if all streams are planned sequentially, and so nearly all the buildings match the lower bound.

- Finally, MIP5 maximizes the residual capacity of rooms. The objective value we found is $\bar{z}_{5 b}=553.74$.

The resulting plan is given below in table 4-8. Each table corresponds to one building, respectively $\mathrm{CW}, \mathrm{BT}, \mathrm{BM}, \mathrm{WE}$ and L. In each table, the first column shows the 11 (out of 15) time slots that are used. Next all the rooms in the building are listed, with the size of the room given above the column. For each time slot and each room, the index of the stream which is placed in the room is given, and in brackets, the estimated number of attendees to this stream. Some rooms have been blocked to be used for purposes other than hosting streams, e.g. the Aula Magna (room 1, building CW) with 665 seats and one of the rooms with 200 seats in building CW (see table 4). In building BT it can be seen that that stream 62 is responsible for the only "hole" in the streams (see table 5). Such holes are the result of additional constraints manually added to the models to account for special scheduling needs such as, the late arrival and early departure of speakers involved in various sessions.

After the EURO2016 conference, delegates were asked to fill out a questionnaire about their satisfaction. Although no question was directly ásked about the schedule of the program, some related questions can give us a hint about the user experience. The question building accessibility (ease of moving around) received a score of 4.6 on a scale from 0 to 5 (with 5 being the highest score). The question overall satisfaction received a score of 4.4 on the same scale. Both questions indicate that the program was logically organized and that related topics were located close to each other so that it was easy to move between talks and sessions.

Another important contribution of the optimization model was that it made it possible to schedule the program much faster than previous EURO-k conferences, which relied on manual or semi-manual scheduling. Having the program available in due time before the conference, is easien for the delegates to plan their trip and decide which sessions and talks to follow. Moreover, there was plenty of time to make minor adjustments to the program.

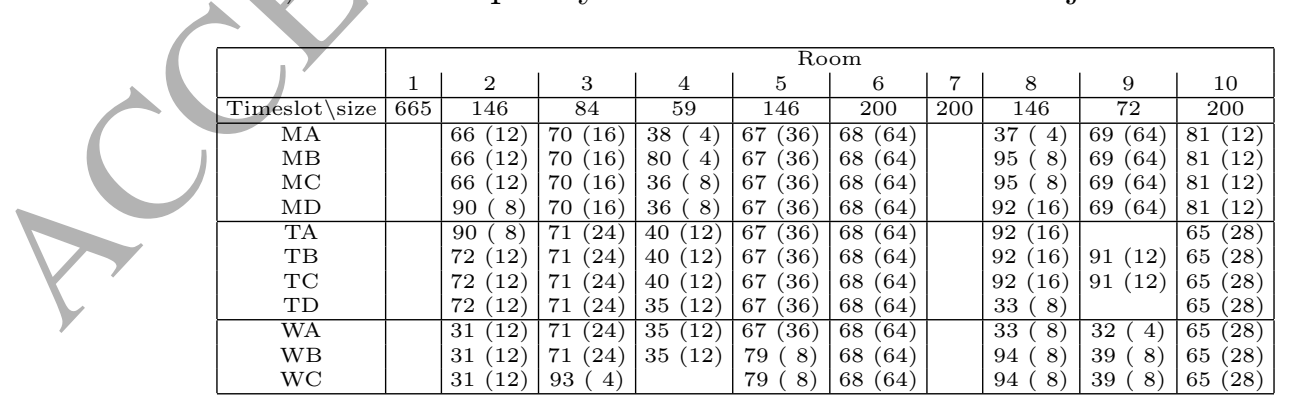

Table 4: Building CW: Allocation of streams to rooms. Each entry reports the stream number and demanded number of seats (in parenthesis). 


\begin{tabular}{|c|c|c|c|c|c|c|c|c|c|c|c|c|c|}
\hline & \multicolumn{13}{|c|}{ Room } \\
\hline & 1 & 2 & 3 & 4 & 5 & 6 & 7 & 8 & 9 & 10 & 11 & 12 & 13 \\
\hline Timeslot $\backslash$ size & 60 & 81 & 96 & 60 & 30 & 40 & 46 & 48 & 76 & 54 & 55 & 64 & 33 \\
\hline MA & $30(28)$ & $110(36)$ & $104(8)$ & $114(20)$ & & $73(16)$ & $29(12)$ & $102(44)$ & $27(16)$ & $28(24)$ & $26(44)$ & $113(44)$ & $1(24)$ \\
\hline MB & $30(28)$ & $110(36)$ & $104(8)$ & $114(20)$ & $61(12)$ & $73(16)$ & $29(12)$ & $102(44)$ & $27(16)$ & $28(24)$ & $26(44)$ & $113(44)$ & $1(24)$ \\
\hline $\mathrm{MC}$ & $30(28)$ & $110(36)$ & $107(32)$ & $114(20)$ & $61(12)$ & $73(16)$ & $29(12)$ & $102(44)$ & $27(16)$ & $28(24)$ & $26(44)$ & $113(44)$ & $1(24)$ \\
\hline MD & $30(28)$ & $110(36)$ & $107(32)$ & $114(20)$ & $61(12)$ & $73(16)$ & $103(16)$ & $102(44)$ & $27(16)$ & $28(24)$ & $26(44)$ & $113(44)$ & $1(24)$ \\
\hline MA & $7(8)$ & $104(8)$ & $113(64)$ & & & $29(12)$ & $111(16)$ & $102(44)$ & $101(4)$ & $108(20)$ & $110(36)$ & $26(60)$ & $1(24)$ \\
\hline MB & $7(8)$ & $104(8)$ & $113(64)$ & $30(28)$ & $62(20)$ & $29(12)$ & $111(16)$ & $102(44)$ & $101(4)$ & $108(20)$ & $110(36)$ & $26(60)$ & $1(24)$ \\
\hline $\mathrm{MC}$ & $28(24)$ & $114(64)$ & $113(64)$ & $30(28)$ & & $29(12)$ & $111(16)$ & $102(44)$ & $101(4)$ & $108(20)$ & $110(36)$ & $26(60)$ & $1(24)$ \\
\hline MD & $28(24)$ & $114(64)$ & $113(64)$ & $30(28)$ & & $107(32)$ & $111(16)$ & $102(44)$ & $63(16)$ & $108(20)$ & $110(36)$ & $26(60)$ & $1(24)$ \\
\hline TA & $28(24)$ & $114(64)$ & $113(64)$ & $30(28)$ & $62(20)$ & $107(32)$ & $100(24)$ & $102(44)$ & $63(16)$ & $108(20)$ & $110(36)$ & $26(60)$ & $1(24)$ \\
\hline TB & $28(24)$ & $114(64)$ & $113(64)$ & $30(28)$ & $62(20)$ & $107(32)$ & $100(24)$ & $102(44)$ & $63(16)$ & & $110(36)$ & $26(60)$ & $1(24)$ \\
\hline TC & $28(24)$ & $114(64)$ & $113(64)$ & $30(28)$ & $62(20)$ & $107(32)$ & $100(24)$ & $102(44)$ & $61(12)$ & & $110(36)$ & $26(60)$ & $106(20)$ \\
\hline $\mathrm{TD}$ & $28(24)$ & $27(60)$ & $113(64)$ & $109(16)$ & $62(20)$ & $107(32)$ & $100(24)$ & $102(44)$ & $61(12)$ & $99(16)$ & $110(36)$ & $26(60)$ & $106(20)$ \\
\hline WA & & $27(60)$ & $113(64)$ & $109(16)$ & $103(16)$ & $107(32)$ & $100(24)$ & $6(12)$ & \begin{tabular}{|l|}
$61(12)$ \\
\end{tabular} & $99(16)$ & $110(36)$ & $26(60)$ & \begin{tabular}{|l|}
$106(20)$ \\
\end{tabular} \\
\hline WB & $8(8)$ & $27(60)$ & $113(64)$ & $109(16)$ & $103(16)$ & $107(32)$ & $100(24)$ & $6(12)$ & $112(8)$ & $99(16)$ & & & $106(20)$ \\
\hline WC & $8(8)$ & $27(60)$ & $113(64)$ & 109 (16) & $103(16)$ & $107(32)$ & & $6(12)$ & $112(8)$ & 99 (16) & & $26(60$ & $106(20)$ \\
\hline
\end{tabular}

Table 5: Building BT: Allocation of streams to rooms. Each entry reports the stream number and demanded number of seats (in parenthesis).

\begin{tabular}{|c|c|c|c|c|c|c|c|c|c|c|c|c|c|c|c|}
\hline & \multicolumn{15}{|c|}{ Room } \\
\hline & 1 & 2 & 3 & 4 & 5 & 6 & 7 & 8 & 9 & 10 & 11 & 12 & 13 & $\mid 14$ & 15 \\
\hline Timeslot \size & 60 & 60 & 30 & 60 & 40 & 40 & 66 & 35 & 16 & 40 & 60 & 60 & 60 & 15 & 30 \\
\hline MA & $97(16)$ & $123(44)$ & $17(16)$ & \begin{tabular}{|l|}
$18(12)$ \\
\end{tabular} & & $84(8)$ & $21(16)$ & $88(24)$ & & $3(28)$ & $119(32)$ & $82(48)$ & \begin{tabular}{|l|}
$78(4)$ \\
\end{tabular} & & $76(8)$ \\
\hline MB & $97(16)$ & $123(44)$ & $17(16)$ & $18(12)$ & & $84(8)$ & $21(16)$ & $88(24)$ & & $3(28)$ & $119(32)$ & $82(48)$ & $89(12)$ & & $76(8)$ \\
\hline $\mathrm{MC}$ & $97(16)$ & $123(44)$ & $17(16)$ & $18(12)$ & & $86(8)$ & $21(16)$ & $88(24)$ & & $3(28)$ & $119(32)$ & $82(48)$ & $89(12)$ & & $98(4)$ \\
\hline MD & $97(16)$ & $123(44)$ & $17(16)$ & $16(8)$ & & $86(8)$ & $21(16)$ & $88(24)$ & & $3(28)$ & $119(32)$ & $82(48)$ & $89(12)$ & & $85(12)$ \\
\hline TA & $115(28)$ & $123(44)$ & $2(28)$ & $16(8)$ & & & $20(8)$ & $88(24)$ & & $3(28)$ & $119(32)$ & $82(48)$ & $89(12)$ & & $85(12)$ \\
\hline $\mathrm{TB}$ & $115(28)$ & $123(44)$ & $2(28)$ & $4(4)$ & & $124(12)$ & $20(8)$ & $88(24)$ & & $3(28)$ & $119(32)$ & $82(48)$ & $5(24)$ & & $85(12)$ \\
\hline $\mathrm{TC}$ & $115(28)$ & $123(44)$ & $2(28)$ & $96(12)$ & & $124(12)$ & $87(8)$ & $75(20)$ & & $3(28)$ & $119(32)$ & $82(48)$ & $5(24)$ & & \\
\hline $\mathrm{TD}$ & $115(28)$ & $123(44)$ & $2(28)$ & $96(12)$ & & $124(12)$ & $87(8)$ & $75(20)$ & $\theta$ & $118(16)$ & $119(32)$ & $82(48)$ & $5(24)$ & & $117(16)$ \\
\hline WA & $115(28)$ & $123(44)$ & $2(28)$ & $96(12)$ & & $120(12)$ & & $75(20)$ & & $118(16)$ & $116(12)$ & $82(48)$ & $5(24)$ & & $117(16)$ \\
\hline WB & $115(28)$ & $123(44)$ & $2(28)$ & $77(8)$ & & $120(12)$ & $19(8)$ & $75(20)$ & & $118(16)$ & $116(12)$ & $82(48)$ & $5(24)$ & & $117(16)$ \\
\hline $\mathrm{WC}$ & $115(28)$ & $83(48)$ & $2(28)$ & $77(8)$ & & $120(12)$ & $19(8)$ & $75(20)$ & & $118(16)$ & $116(12)$ & $82(48)$ & $5(24)$ & & $117(16)$ \\
\hline
\end{tabular}

Table 6: Building BM: Allocation of streams to rooms. Each entry reports the stream number and demanded number of seats (in parenthesis).

\begin{tabular}{|c|c|c|c|c|c|c|c|c|c|c|c|c|}
\hline & \multicolumn{12}{|c|}{ Room } \\
\hline & 1 & & 3 & 4 & 5 & 6 & 7 & 8 & 9 & 10 & 11 & 12 \\
\hline Timeslot \size & 42 & 42 & 90 & 60 & 30 & 90 & 90 & 40 & 36 & 60 & 90 & 24 \\
\hline $\mathrm{MA}$ & $41(8)$ & $50(20)$ & $51(28)$ & $58(16)$ & $46(12)$ & & & & $122(20)$ & $25(4)$ & & \\
\hline MB & $49(8)$ & $50(20)$ & $51(28)$ & $58(16)$ & $46(12)$ & & & & $122(20)$ & $55(4)$ & & \\
\hline $\mathrm{MC}$ & 49 (8) & $50(20)$ & $51(28)$ & $58(16)$ & $46(12)$ & & & & $122(20)$ & $52(4)$ & & \\
\hline MD & $105(8)$ & $50(20)$ & $51(28)$ & $58(16)$ & $44(12)$ & & & & $122(20)$ & $48(12)$ & & \\
\hline $\mathrm{TA}$ & $105(8)$ & $59(8)$ & $51(28)$ & $56(4)$ & $44(12)$ & & & & $122(20)$ & $48(12)$ & & \\
\hline $\mathrm{TB}$ & $23(16)$ & $59(8)$ & $51(28)$ & $43(16)$ & $44(12)$ & & & & $53(4)$ & $48(12)$ & & \\
\hline $\mathrm{TC}$ & $23(16)$ & $57(8)$ & $51(28)$ & $43(16)$ & $22(20)$ & & & & $121(20)$ & $42(20)$ & & \\
\hline $\mathrm{TD}$ & $23(16)$ & $57(8)$ & $45(8)$ & $43(16)$ & $22(20)$ & & & & $121(20)$ & $42(20)$ & & \\
\hline WA & $23(16)$ & $47(12)$ & $45(8)$ & $43(16)$ & $22(20)$ & & & & $121(20)$ & $42(20)$ & & \\
\hline & $60(8)$ & $47(12)$ & $24(8)$ & $54(8)$ & $22(20)$ & & & & $121(20)$ & $42(20)$ & & \\
\hline & $60(8)$ & $47(12)$ & $24(8)$ & $54(8)$ & $22(20)$ & & & & $121(20)$ & $42(20)$ & & \\
\hline
\end{tabular}

Table 7: Building WE: Allocation of streams to rooms. Each entry reports the stream number and demanded number of seats (in parenthesis).

\subsection{A note on multi-objective optimization}

The conference scheduling approach described here relies on the lexicographic optimization of five different objectives. This is normally not a good approach when facing a multiobjective problem. However, in our case, we can compare the first four objectives with a corresponding bound, and for each of them we can see that we are not too far from the optimal solution. Hence, the stepwise approach has not introduced much deviation. A better approach would be to simultaneously optimizing all five objectives, find the entire Pareto 


\begin{tabular}{|c|c|c|c|c|}
\hline & \multicolumn{4}{|c|}{ Room } \\
& 1 & 2 & 3 & 4 \\
\hline Timeslot \size & 126 & 120 & 155 & 222 \\
\hline MA & $13(16)$ & & $73(16)$ & $9(48)$ \\
MB & $13(16)$ & $11(8)$ & $73(16)$ & $9(48)$ \\
MC & $13(16)$ & $11(8)$ & $73(16)$ & $9(48)$ \\
MD & $13(16)$ & $12(8)$ & $73(16)$ & $9(48)$ \\
\hline TA & & $12(8)$ & $74(4)$ & $9(48)$ \\
TB & $14(16)$ & $15(16)$ & $64(24)$ & $9(48)$ \\
TC & $14(16)$ & $15(16)$ & $64(24)$ & $9(48)$ \\
TD & $14(16)$ & $15(16)$ & $64(24)$ & $9(48)$ \\
\hline WA & $14(16)$ & $15(16)$ & $64(24)$ & $9(48)$ \\
WB & & & $64(24)$ & $9(48)$ \\
WC & $10(48)$ & & $64(24)$ & $9(48)$ \\
\hline
\end{tabular}

Table 8: Building L: Allocation of streams to rooms. Each entry reports the stream number and demanded number of seats (in parenthesis).

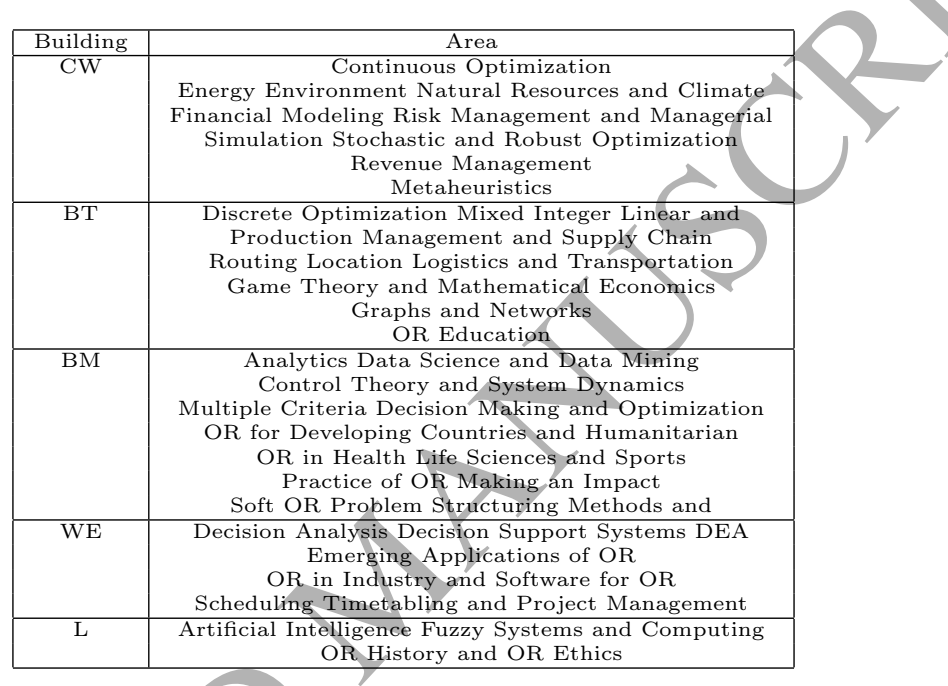

Table 9: The distribution of areas in the different buildings

Optimal front and select the solution with the best trade-offs. Despite recent improvements in multi-objective optimization, see e.g. [7, 8, 9], such an approach is not realistic using current techniques. For a good introduction to multi-objective optimization we refer to [10].

\section{Challenges and future improvements}

We consider the approach successfull, although we cannot quantify it by more than anecdotal evidence: The conference leadership seems to have received fewer complains and the whole process of scheduling were a much more relaxed experience, where the usual allnight sessions of people manually typing the plan into the database was missing. That said, there are a couple of things we think can be improved.

In the model, we need to get an estimate of the number of attendees to each stream. This number is today simply the number of presenters in the stream, relying on the assumption that all presenters in a stream will participate in all sessions of the stream they are presenting in. We acknowledge that this estimation of stream attendee numbers is rather weak. The 
quality of the plan could probably be improved with a better estimate. It is, however, not clear to us how to obtain this.

The second problem is that we have no information about which stream each participant wants to attend, hence we do not know if certain sessions preferably should not be placed in the same time slots.

Both the above mentioned problems could be solved in the conference registration process, by requiring information on which streams the registrants plan to attend.Moreover, it is difficult for the participant to decide which streams to follow without having seen the program. In addition, it is important to not make the registration process too complicated.

Finally, avoidance of clash of co-authors could be supported in the scheduling process. This is however complicated by the fact that the detailed plan for the stream is made by the stream organizer.

\section{Conclusion}

The tool made it possible to schedule the program in due time before the conference. Most delegates were very satisfied with the schedule and found it easy to navigate through the conference. The tool was also used to make the schedule for IFORS 2017 in Quebec City and it is planned to be used for both EURO 2018 in Valencia and for IFORS 2020 Seoul.

Future developments of the optimization tool could include better optimization of room sizes and to avoid conflicts between popular talks. However, this would demand better information about what the delegates wish to hear.

We would also like to include some fairness in the schedule, so that sessions with a very low attendance are avoided. This could be achieved by having at least one popular talk in each session. However this is difficult to achieve without breaking the hierarchical structure of the conference.

In the future, EURO could possibly use this tool to evaluate whether or not university bids for the EURO conference are feasible. This test could verify that there is indeed enough rooms for the schedule, e.g. by simply scheduling the previous EURO conference given the buildings and rooms available at the bidding university.

Finally, we hope that this article can contribute to increased transparency of the scheduling process for the EURO conferences, as well as inspire the scheduling of other conferences.

\section{References}

[1] B. Vangerven, A. Ficker, D. Goossens, W. Passchyn, F. Spieksma, G. J. Woeginger, Conference scheduling - a personalized approach, in: EURO-2016 Conference program, 2016.

[2] A. Bhardwaj, J. Kim, S. Dow, D. Karger, S. Madden, R. Miller, H. Zhang, Attendee-sourcing: Exploring the design space of community-informed conference scheduling, in: Second AAAI Conference on Human Computation and Crowdsourcing, 2014.

[3] A. X. Zhang, A. Bhardwaj, D. Karger, Confer: A conference recommendation and meetup tool, in: CSCW '16 Companion Proceedings of the 19th ACM Conference on Computer Supported Cooperative Work and Social Computing Companion, 2016, pp. 118-121.

[4] S. E. Sampson, Practical implications of preference-based conference scheduling, Production and Operations Management 13.3 (Fall 2004) (2004) 205-215. 
[5] J. Kim, H. Zhang, P. Andr, L. B. Chilton, W. Mackay, M. Beaudouin-Lafon, R. C. Miller, S. P. Dow, Cobi: a community-informed conference scheduling tool, in: UIST '13 Proceedings of the 26th annual ACM symposium on User interface software and technology, ACM New York, NY, USA, 2013, pp. 173-182.

[6] T. Stidsen, D. Pisinger, D. Vigo, Conference scheduling data for euro-2016 in poznan (2017).

URL http://www.moretime.man.dtu.dk/

[7] P. Belotti, B. Soylu, M. Wiecek, A branch-and-bound algorithm for biobjective mixed-integer programs, Optimization Online, optimization-online.org.

[8] N. Boland, H. Charkhgard, M. Savelsbergh, The l-shape search method for triobjective integer programming, Mathematical Programming Computation 8 (2) (2016) 217-251. doi:10.1007/s12532-015-0093-3. URL http://dx.doi.org/10.1007/s12532-015-0093-3

[9] T. R. Stidsen, K. A. Andersen, B. Dammann, A branch and bound algorithm for a class of biobjective mixed integer programs, Management Science 60 (4) (2014) 1009-1032. doi:10.1287/mnsc.2013.1802.

[10] M. Ehrgott, Multicriteria Optimization, Springer Verlag, Berlin, 2nd edition, 2005.

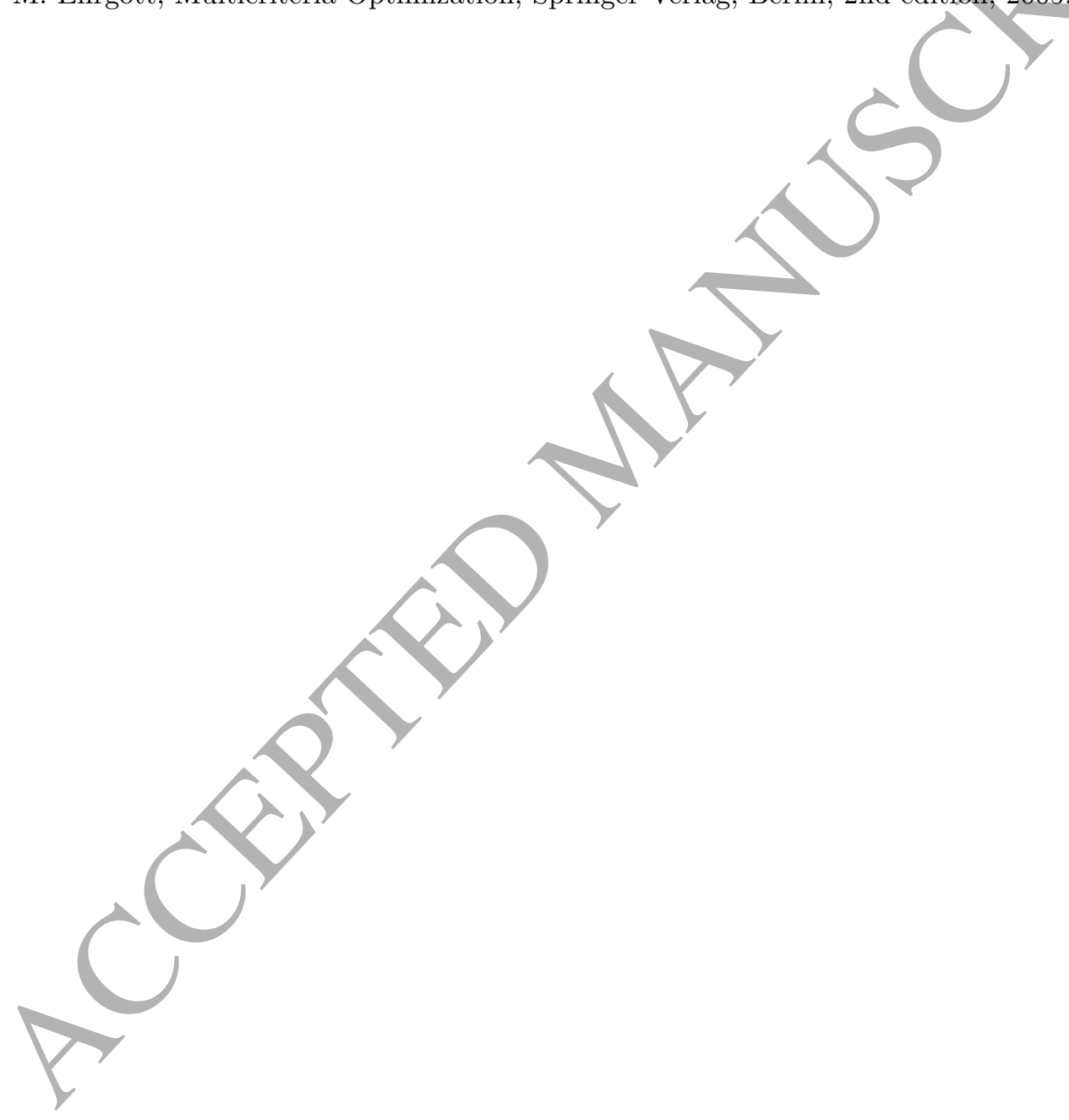

